\title{
Predictors of unfavorable outcomes following deep brain stimulation for movement disorders and the effect of hospital case volume on outcomes: an analysis of 33,642 patients across 234 US hospitals using the National (Nationwide) Inpatient Sample from 2002 to 2011
}

\author{
Piyush Kalakoti, MD, Osama Ahmed, MD, Papireddy Bollam, MD, Symeon Missios, MD, \\ Jessica Wilden, MD, and Anil Nanda, MD, MPH \\ Department of Neurosurgery, Louisiana State University Health Sciences Center, Shreveport, Louisiana
}

\begin{abstract}
OBJECT With limited data available on association of risk factors and effect of hospital case volume on outcomes following deep brain stimulation (DBS), the authors attempted to identify these associations using a large population-based database.

METHODS The authors performed a retrospective cohort study involving patients who underwent DBS for 3 primary movement disorders: Parkinson's disease, essential tremor, and dystonia from 2002 to 2011 using the National (Nationwide) Inpatient Sample (NIS) database. Using national estimates, the authors identified associations of patient demographics, clinical characteristics, and hospital characteristics on short-term postoperative outcomes following DBS. Additionally, effect of hospital volume on unfavorable outcomes was investigated.
\end{abstract}

RESULTS Overall, 33,642 patients underwent DBS for 3 primary movement disorders across 234 hospitals in the US. The mean age of the cohort was $63.42 \pm 11.31$ years and $36 \%$ of patients were female. The inpatients' postoperative risks were $5.9 \%$ for unfavorable discharge, $10.2 \%$ for prolonged length of stay, $14.6 \%$ for high-end hospital charges, $0.5 \%$ for wound complications, $0.4 \%$ for cardiac complications, $1.8 \%$ for venous thromboembolism, and $5.5 \%$ for neurological complications, including those arising from an implanted nervous system device. Compared with low-volume centers, odds of having an unfavorable discharge, prolonged LOS, high-end hospital charges, wound, and cardiac complications were significantly lower in the high-volume and medium-volume centers.

CONCLUSIONS The authors' study provides individualized estimates of the risks of postoperative complications based on patient demographics and comorbidities and hospital characteristics, which could potentially be used as an adjunct for risk stratification for patients undergoing DBS.

http://thejns.org/doi/abs/10.3171/2015.3.FOCUS1547

KEY WORDS deep brain stimulation; movement disorders; unfavorable outcomes; National (Nationwide) Inpatient Sample

$\mathrm{D}$ EEP brain stimulation (DBS) refers to the delivery of pulsed, high-frequency electrical current to subcortical structures to improve disease symptoms. ${ }^{17}$ Although DBS emerged as a potential substitute to lesioning in the late 1960 s to control severe, chronic, intractable pain, ${ }^{22}$ its application in movement disorders was not reported until 1987.6,39 After DBS was successfully applied to the thalamic ventral intermediate nuclei to suppress intractable tremor as demonstrated independently by Benabid and colleagues ${ }^{5-7}$ and Siegfried and Shulman, ${ }^{39}$ its utility has been explored in treating various movement disorders. In 1997, the FDA approved DBS of the unilateral thalamus for the treatment of essential tremor and Parkinson's disease tremor. In 2002, approval was granted

ABBREVIATIONS AUC = area under the curve; $\mathrm{CCI}=$ Charlson Comorbidity Index; $\mathrm{CRF}=$ chronic renal failure; $\mathrm{DBS}=$ deep brain stimulation; $\mathrm{DM}=$ diabetes mellitus; HCUP = Healthcare Cost and Utilization Project; HVC = high-volume center; LOS = length of stay; LVC = low-volume center; MVC = medium-volume center; NIS = National (Nationwide) Inpatient Sample; PD = Parkinson's disease; ROC = receiver operating characteristic.

SUBMITTED February 2, 2015. ACCEPTED March 25, 2015.

INCLUDE WHEN CITING DOI: 10.3171/2015.3.FOCUS1547.

DISCLOSURE The authors report no conflict of interest concerning the materials or methods used in this study or the findings specified in this paper. 
for DBS of the subthalamic nucleus and globus pallidus internus for PD treatment, and in 2003, on the basis of the Humanitarian Device Exemption, approval was expanded to include treatment of primary dystonia. ${ }^{17}$ Since then, DBS has gained nationwide popularity.

Several studies have investigated long-term outcomes in patients undergoing DBS..$^{5,9,12,23,24,27,31,33,34}$ Most of these studies are retrospective analyses of a single institutional experience, limiting the ability to generalize conclusions owing to inherent selection bias. Previous multicenter studies also have inherent limitations given their focus on a particular region and the small number of centers. Most of these studies examine long-term outcomes following DBS rather than immediate or short-term postoperative outcomes encountered during in-hospital stay. To examine DBS outcomes from a large sample size and a diversity of practice settings, we used the National (Nationwide) Inpatient Sample (NIS), a prospective hospital discharge database representing a random, validated sample of all inpatient admissions to nonfederal hospitals in the US. We identified independent predictors associated with adverse short-term outcomes in patients undergoing DBS procedures for 3 primary movement disorders: essential tremor, $\mathrm{PD}$, and dystonia. Additionally, we investigated the effect of hospital caseload volume on outcomes following DBS. To our knowledge, this study provides the latest follow-up in DBS utilizing the NIS database.

\section{Methods \\ Data Source}

The NIS, an element of the Healthcare Cost and Utilization Project (HCUP), was the data source for this study. ${ }^{42}$ With nearly 1000 hospitals participating in the HCUP, the NIS is considered the largest publicly available inpatient all-payer database in the US. Comprising data resulting from more than 7 million inpatient hospital admissions, the NIS represents a $20 \%$ stratified subsample of all nonfederal US hospitals ${ }^{2}$ and permits the assessment of national estimates using discharge-level weights. Using the Clinical Classifications Software (CCS) coding system as developed by the Agency for Healthcare Research and Quality (AHRQ; Rockville, Maryland), the inpatient data in the NIS is sequenced into clinically relevant categories comprising 14,000 diagnoses and 3900 procedures as adapted from the ICD-9-CM codes. ${ }^{1}$ Further information about the database can be accessed at http://www.hcup-us. ahrq.gov/nisoverview.jsp.

\section{Cohort Definition}

Having previously compiled the NIS data for the years 2002 to 2011 onto a single server using statistical software (version 22.0, SPSS, IBM), an inquiry was initiated for identification of patients (older than 18 years) undergoing a DBS procedure (ICD-9-CM procedure code 02.93 ) for any of the 3 primary movement disorders: PD (ICD-9-CM diagnosis code 332.0,332.1), essential tremor (ICD-9-CM diagnosis code 333.1), and dystonia (ICD-9-CM 333.6-333.8, $333.71,333.72,333.79,333.81-333.85,333.89)$ (Fig. 1).

\section{Patient and Hospital Characteristics}

Patient characteristics cataloged in the NIS include age, sex, race, primary payer, median household income for ZIP code of residence (described as quartiles with reference to US national values), number of presenting diagnoses, and number of procedures. To evade aberrant coefficients in the regression models, infinitesimal categories for certain exposure variables were compounded. Native American patients $(0.2 \%)$ and those with payment source of "no charge" $(0.1 \%)$ were designated into "other" race and "other" payment source, respectively. To quantify the effect of patient comorbidities, stratification of medical comorbidity was achieved using the Charlson Comorbidity Index (CCI) ${ }^{13}$ as revised by Deyo et al. ${ }^{15}$ to use on ICD-9-CM codes. ${ }^{37}$

The hospital characteristics coded in the NIS include region, bedsize, and teaching status. Additionally, each case registered in the NIS is assigned an encrypted HCUP hospital identifier number. To evaluate patient demographics and outcomes across centers with variable volume caseloads, we used this variable to compute number of DBS procedures performed by each hospital $(n=234)$ over time (Fig. 2) and established 3 categories of hospital volumes: high-volume centers (HVCs), medium-volume centers (MVCs), and low-volume centers (LVCs). Subsequently, cases were clustered as being performed at an LVC (103 hospitals) if the hospital performed 1-49 DBS procedures $(<5$ per year), MVC (113 hospitals) if there were $50-449$ DBS procedures ( $\geq 5$ and $\leq 45$, per year), and HVC (18 hospitals) if there were $\geq 450$ DBS procedures ( $\geq 45$ per year) performed over the study period. These volume assignments and cutoff values were selected preferentially based on our experience.

\section{Outcome Variables}

Primary outcome variables were unfavorable discharge, prolonged length of stay (LOS), high-end hospital charges, and short-term postoperative complications: wound complications, cardiac complications, venous thromboembolism, and neurological complications. Nominal variables with more than 2 levels of categories (discharge disposition) and continuous variables of interest (LOS and hospital charges) were dichotomized. Discharge disposition was dichotomized into routine (routine discharge and home health care) or unfavorable (transfer to short-term hospital, skilled nursing facility, intermediate care, or rehabilitation). Patients with LOS above the 90th percentile of the total number of days from admission to discharge were defined as having a "prolonged LOS," and patients having incurred hospital charges above the 90th percentile during the course of inpatient stay were labeled as having "high-end hospital charges." Short-term postoperative complications were defined as those encountered during the course of inpatient hospitalization following the surgical procedure. For all calculations pertaining to hospital charges, inflation adjustment for each year over the 10year study period was done to the fiscal year 2013 US dollar value using the consumer price index calculator. ${ }^{11}$ Following identification of independent predictors for each of the above-described outcome variables, a comparison of unfavorable outcomes between HVCs and LVCs, and between MVCs and LVCs was performed.

Secondary outcome included estimation of number of 


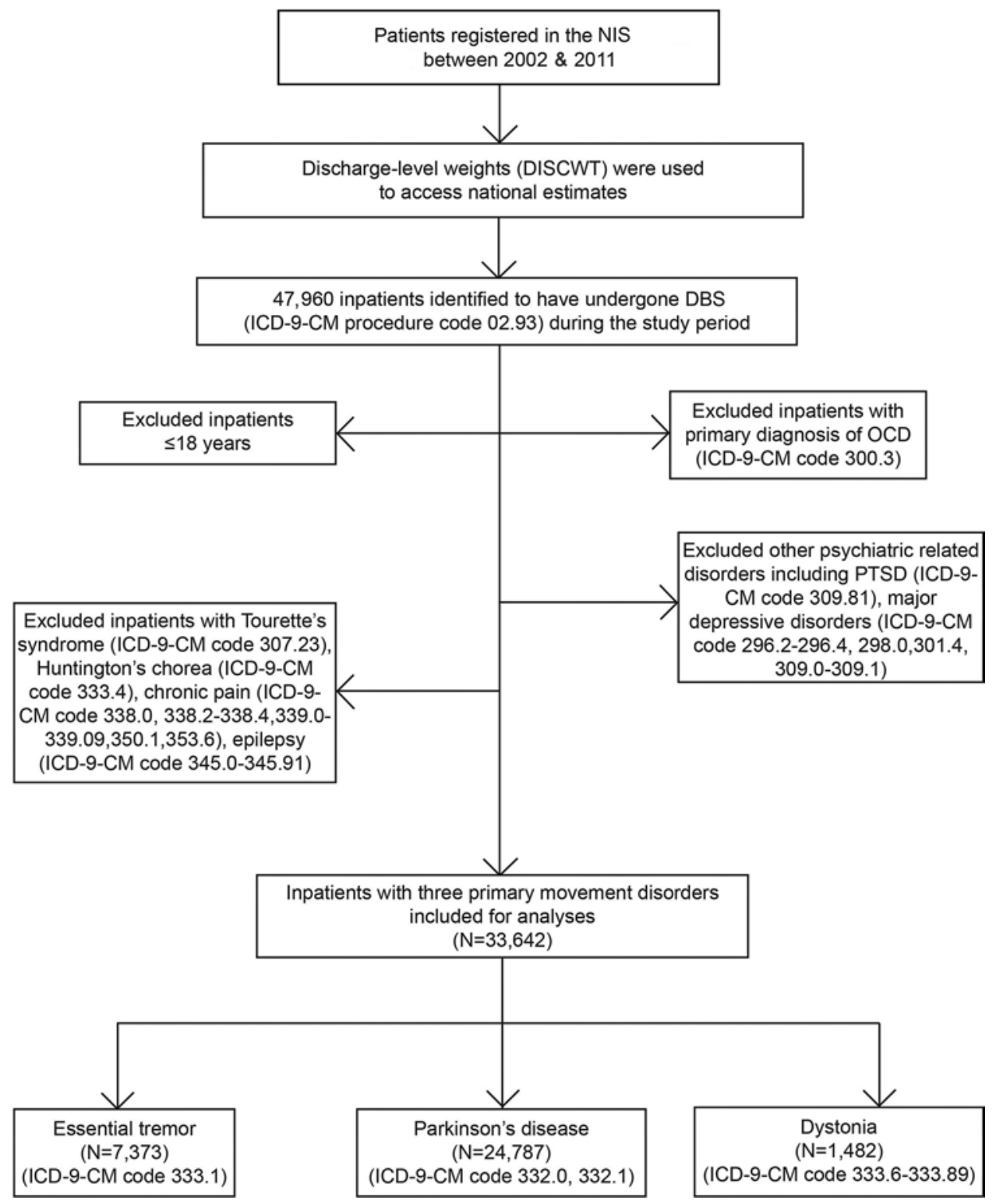

FIG. 1. Algorithm of cohort selection for the study. $O C D=$ obsessive-compulsive disorder; $P T S D=$ posttraumatic stress disorder.

DBS procedures performed per year over time and estimation of annual rates of DBS procedures performed per 100,000 US population over time. National estimates of number of DBS procedures per year over time were used, and data provided by the US Census Bureau, Population Division (December 2011) (3,44 $^{4,}$ were used to calculate annual rates of DBS. Lastly, trends of incurred hospital charges over time between HVC and LVC were examined for patients undergoing DBS in the US.

\section{Statistical Analysis}

All statistical analyses were conducted using commercially available SPSS (version 22.0, IBM) and XLSTAT (version 2014.5.03, Addinsoft). All data presented correspond to national estimates using the discharge-level weights in the NIS. The data were analyzed for descriptive and inferential statistics. Descriptive statistics were used to examine the patient and hospital characteristics. For continuous variables, mean \pm SD or median are reported as appropriate with comparison of means using 1-way ANOVA. Categorical values are depicted as numbers and/ or percentages and were compared using the chi-square test. The Pearson correlation coefficient was used to assess the degree of linear relationship between the numbers of DBS cases performed and incurred hospital charges over time.

To identify independent associations of variables with defined outcomes, a multivariable binary logistic regression analysis using the forward conditional method was performed while adjusting for age, sex, race (African American, Hispanic, Asian, or other, with Caucasians being the reference value), median income quartile (second, third, or fourth, with reference to first income quartile), payer (Medicaid, private including HMO, self-payer, or other with Medicare being the reference), patient comorbidities, type of movement disorder (PD or dystonia, with 


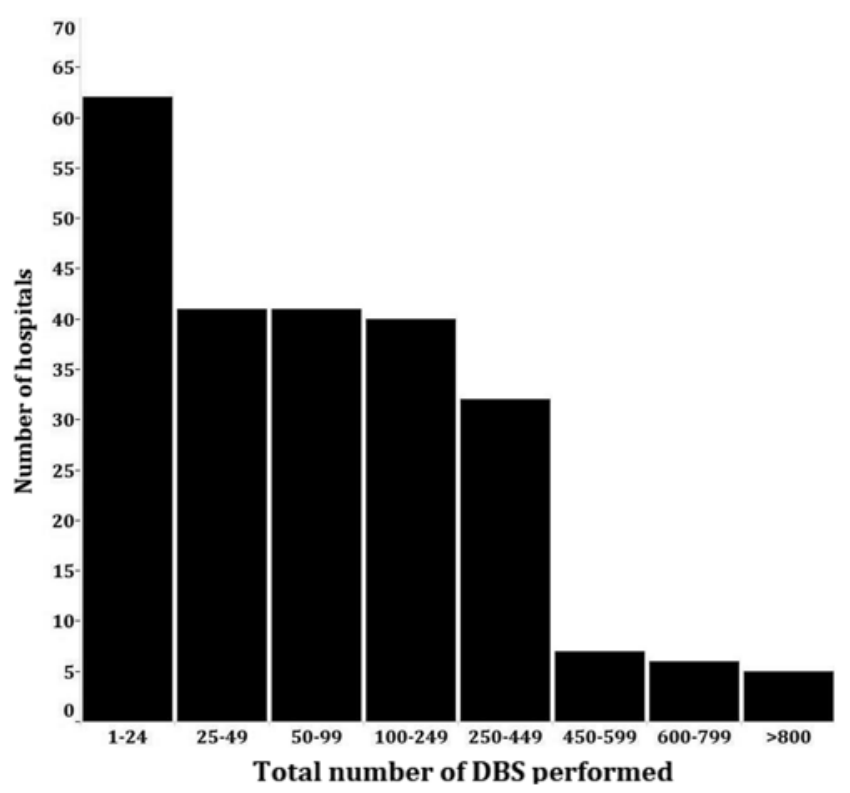

FIG. 2. National estimates of distribution of total number of DBS procedures performed in the US over the study period (2002-2011) by number of hospitals $(n=234)$.

essential tremor being the reference value), number of presenting diagnoses, number of procedures during inpatient stay, hospital characteristics including region (West, South, Midwest, with Northeast being the reference), teaching status (urban nonteaching or urban teaching, with rural being the reference), bedsize (medium or large, with small being the reference value), and volume load centers as defined above (HVC or MVC, with LVC being the reference value). Age, number of presenting diagnoses, and number of procedures were continuous variables. Sex, race, income quartiles, insurance status, type of movement disorder, and hospital characteristics were categorical variables. The patient-level comorbidities (categorical variables) were hypertension, coronary artery disease, chronic pulmonary disease, hypercholesterolemia, coagulopathy, diabetes mellitus (DM), previous history of ischemic stroke, seizures, peripheral vascular disease, obesity, chronic renal failure (CRF), obesity, alcohol abuse, and tobacco exposure. In addition, a comparison of outcomes between HVCs and MVCs, each in reference to LVCs, was performed.

For variables adjusted in the regression model, multiple imputation was performed for missing values after estimating the proportions of missing values. These included age $(1.2 \%)$, sex $(1.6 \%)$, race $(19.0 \%)$, payer $(0.2 \%)$, income $(2.7 \%)$, hospital bedsize (1.3\%), and teaching status $(1.3 \%)$. Observation of patterns of missing variables suggested the missing to be random. Imputation was performed in SPSS for missing data on the basis of existing variables. The iteration was performed 5 times, creating 5 imputed data sets that were combined to create a pooled data set to be used in the regression model with no subsequent missing values. Regression diagnostics was performed for all logistic regression models. $\mathrm{C}$-index statistics or the area under the receiver operating characteristic (ROC) curve was used to assess the discriminatory ability of the regression model.
Depicting a graphical representation of the sensitivity (y axis) over 1 minus specificity (x axis), a perfect model or test has a sensitivity of 1 , a 1 minus specificity as 0 , and an area under the curve (AUC) as 1 that is represented by a point on the top left corner of the graph suggesting detection of all true positives with no false positives. A value of 0.5 suggests no discriminatory ability at all (not better than chance) and is represented by a diagonal line. Higher values of AUC of ROC curves denote a better discriminating ability of a test or model between a true positive and a false positive value. All statistical tests used were 2 -sided, and $\mathrm{p}<0.05$ was deemed statistically significant.

\section{Results}

\section{Patient Characteristics and Incidence of DBS}

During the selected study period, 33,642 patients underwent DBS for 3 primary movement disorders across 234 hospitals in the US. The mean age of the cohort was $63.42 \pm 11.31$ years and $36 \%$ of patients were female. The distribution of the demographics and clinical characteristics of patients across various volume centers are depicted in Table 1.

\section{Clinical Outcomes}

The overall postoperative risks were $5.9 \%$ for unfavorable discharge, $10.2 \%$ for prolonged LOS, $14.6 \%$ for highend hospital charges, $0.5 \%$ for wound complications, $0.4 \%$ for cardiac complications, $1.8 \%$ for venous thromboembolism, and $5.5 \%$ for neurological complications, including those arising from an implanted nervous system device. The distribution of these unfavorable outcomes across various hospital case-volume centers is noted in Table 2.

\section{Regression Model Derivation}

A multivariable binary regression analysis investigating the effect of independent variables on the likelihood of unfavorable discharge (Fig. 3A), cardiac complications (Fig. 3B), wound infections (Fig. 3C), and venous thromboembolism (Fig. 3D), prolonged LOS (Fig. 4 left) and high-end hospital charges (Fig. 4 right) is depicted in the corresponding forest plots. Following identification of independent risk factors for unfavorable outcomes after DBS, a comparison of these adverse outcomes was made between HVCs and LVCs and between MVCs and LVCs as depicted in Fig. 5 after adjusting for age, sex, race, income quartiles, payer, patient comorbidities, type of movement disorder, number of presenting diagnoses, number of procedures during inpatient stay, and hospital characteristics.

\section{Accuracy of the Model}

The AUC or C statistic of the ROC was used to validate the accuracy of the regressions. The AUC was 0.76, 0.90, $0.88,0.83,0.77$, and 0.75 for unfavorable discharge, cardiac complication, wound complications, venous thromboembolism, prolonged LOS, and high-end hospital charges, respectively.

\section{Secondary Outcomes}

A significant increase by 2.06 -fold in the total num- 
TABLE 1. Demographic and clinical characteristics of 33,642 patients undergoing DBS in the US between 2002 and 2011

\begin{tabular}{|c|c|c|c|c|c|}
\hline Characteristics & Overall & LVCs & MVCs & HVCs & p Value* \\
\hline No. of patients & 33,642 & 1728 & 15,412 & 16,502 & \\
\hline \multicolumn{6}{|l|}{ Demographics } \\
\hline Mean age $\pm S D$ in yrs & $63.42 \pm 11.31$ & $64.73 \pm 9.49$ & $63.75 \pm 11.06$ & $62.97 \pm 11.69$ & $<0.0001$ \\
\hline Female sex, n (\%) & $5782(35.6)$ & $579(33.5)$ & $5468(36.1)$ & $5782(35.6)$ & 0.098 \\
\hline \multicolumn{6}{|l|}{ Race, n (\%) } \\
\hline Caucasian & $23,316(86.7)$ & $1224(83.8)$ & $11,272(88.1)$ & $10,820(85.5)$ & $<0.0001$ \\
\hline African American & $379(1.4)$ & $33(2.3)$ & $211(1.6)$ & $135(1.1)$ & $<0.0001$ \\
\hline Hispanic & $1675(6.2)$ & $37(2.5)$ & $776(6.1)$ & $62(6.8)$ & $<0.0001$ \\
\hline Asian & $551(2.0)$ & $42(2.9)$ & $216(1.7)$ & $293(2.3)$ & $<0.0001$ \\
\hline Other & $980(3.6)$ & $125(8.6)$ & $313(2.4)$ & $542(4.3)$ & $<0.0001$ \\
\hline \multicolumn{6}{|l|}{ Income, n (\%) } \\
\hline Lowest quartile & 5547 (16.9) & $292(17.2)$ & $2820(18.8)$ & $2425(15.3)$ & $<0.0001$ \\
\hline Second quartile & $8068(24.6)$ & $515(30.3)$ & $3823(25.4)$ & $3730(23.2)$ & $<0.0001$ \\
\hline Third quartile & 8887 (27.2) & $512(30.1)$ & $3894(26.0)$ & $4481(27.9)$ & $<0.0001$ \\
\hline Fourth quartile & $10,247(31.3)$ & $381(22.4)$ & $4468(29.8)$ & $5398(33.6)$ & $<0.0001$ \\
\hline \multicolumn{6}{|l|}{ Primary payer, n (\%) } \\
\hline Medicare & $21,016(62.6)$ & $1081(62.9)$ & 9729 (63.2) & $10,206(61.9)$ & 0.055 \\
\hline Medicaid & $881(2.6)$ & $30(1.7)$ & $472(3.1)$ & $379(2.3)$ & $<0.0001$ \\
\hline Private & $10,789(32.1)$ & $583(33.9)$ & $4729(30.7)$ & $5477(33.2)$ & $<0.0001$ \\
\hline Self & $129(0.4)$ & $15(0.9)$ & $68(0.5)$ & $46(0.3)$ & $<0.0001$ \\
\hline Other & $769(2.3)$ & $\leq 10 \dagger$ & $388(2.5)$ & $371(2.3)$ & $<0.0001$ \\
\hline \multicolumn{6}{|l|}{ Teaching status, $n(\%)$} \\
\hline Rural & $581(1.8)$ & $11(0.6)$ & $276(1.8)$ & $294(1.8)$ & 0.001 \\
\hline Urban nonteaching & $4122(12.4)$ & $721(41.7)$ & $1943(13.0)$ & $1458(8.8)$ & $<0.0001$ \\
\hline Urban teaching & $28,490(85.8)$ & $997(57.7)$ & $12,743(85.2)$ & $14,750(89.4)$ & $<0.0001$ \\
\hline \multicolumn{6}{|l|}{ Bedsize, n (\%) } \\
\hline Small & $1997(6.0)$ & $175(10.1)$ & $780(5.2)$ & $1042(6.3)$ & $<0.0001$ \\
\hline Medium & $2682(8.1)$ & $356(20.6)$ & $1933(12.9)$ & $393(2.4)$ & $<0.0001$ \\
\hline Large & $28,514(85.9)$ & $1198(69.3)$ & $12,248(81.9)$ & $15,068(91.3)$ & $<0.0001$ \\
\hline \multicolumn{6}{|l|}{ Region, n (\%) } \\
\hline Northeast & $4476(13.3)$ & $213(12.3)$ & $2429(15.8)$ & $1834(11.1)$ & $<0.0001$ \\
\hline Midwest & $6240(18.5)$ & $576(33.3)$ & $3015(19.6)$ & 2649 (16.1) & $<0.0001$ \\
\hline South & $12,305(36.6)$ & $718(41.6)$ & $5446(35.3)$ & $6141(37.2)$ & $<0.0001$ \\
\hline West & $10,621(31.6)$ & $221(12.8)$ & $4522(29.3)$ & $5878(35.6)$ & $<0.0001$ \\
\hline \multicolumn{6}{|l|}{ Hospital charges, US\$‡ } \\
\hline Mean & 65,500 & 66,063 & 70,357 & 60,399 & $<0.0001$ \\
\hline Median & 54,721 & 49,522 & 58,196 & 53,024 & \\
\hline \multicolumn{6}{|c|}{ Clinical characteristics, n (\%) } \\
\hline Stroke & $336(1.0)$ & $\leq 10 \dagger$ & $136(0.9)$ & $195(1.2)$ & $<0.0001$ \\
\hline CAD & $3495(10.4)$ & $204(11.8)$ & $1629(10.6)$ & $1662(10.1)$ & 0.049 \\
\hline COPD & $2901(8.6)$ & $191(11.0)$ & $1297(8.4)$ & $1413(8.6)$ & 0.001 \\
\hline CRF & $224(0.7)$ & $21(1.2)$ & $77(0.5)$ & $126(0.8)$ & $<0.0001$ \\
\hline Hypercholesterolemia & $5764(17.1)$ & $398(23.0)$ & $2526(16.4)$ & $2840(17.2)$ & $<0.0001$ \\
\hline Smoking & $5527(16.4)$ & $298(17.2)$ & $2245(14.6)$ & $2984(18.1)$ & $<0.0001$ \\
\hline Obesity & $1157(3.4)$ & $48(2.8)$ & $433(2.8)$ & $676(4.1)$ & $<0.0001$ \\
\hline Alcohol abuse & $177(0.5)$ & $\leq 10 \dagger$ & $78(0.5)$ & $94(0.6)$ & 0.277 \\
\hline Coagulopathy & $197(0.6)$ & $14(0.8)$ & $78(0.5)$ & $105(0.6)$ & 0.142 \\
\hline $\mathrm{CHF}$ & $522(1.6)$ & $33(1.9)$ & $261(1.7)$ & $288(1.4)$ & 0.037 \\
\hline
\end{tabular}


TABLE 1. Demographic and clinical characteristics of 33,642 patients undergoing DBS in the US between 2002 and 2011 (continued)

\begin{tabular}{|c|c|c|c|c|c|}
\hline Characteristics & Overall & LVCs & MVCs & HVCs & p Value* \\
\hline \multicolumn{6}{|c|}{$\begin{array}{l}\text { Clinical characteristics, } n(\%) \\
\quad \text { (continued) }\end{array}$} \\
\hline Hypertension & $11,884(35.3)$ & $707(40.9)$ & $5535(35.9)$ & $5642(34.2)$ & $<0.0001$ \\
\hline DM & $3772(11.2)$ & $248(14.3)$ & $1749(11.3)$ & $1775(10.8)$ & $<0.0001$ \\
\hline PVD & $424(1.3)$ & $\leq 10 \dagger$ & $172(1.1)$ & $243(1.5)$ & $<0.0001$ \\
\hline Seizures & $931(2.8)$ & $55(3.2)$ & $388(2.5)$ & $488(3.0)$ & 0.032 \\
\hline \multicolumn{6}{|l|}{$\mathrm{CCl}$} \\
\hline Low (0-3) & $33,465(99.5)$ & $1728(100.0)$ & $15,328(99.5)$ & $16,409(99.4)$ & 0.008 \\
\hline Moderate/high $(\geq 4)$ & $177(0.5)$ & $0(0.0)$ & $84(0.5)$ & $93(0.6)$ & 0.008 \\
\hline \multicolumn{6}{|l|}{ No. of diagnoses } \\
\hline Mean & 4.42 & 4.32 & 4.36 & 4.48 & $<0.0001$ \\
\hline Median & 4 & 4 & 4 & 4 & \\
\hline \multicolumn{6}{|l|}{ No. of procedures } \\
\hline Mean & 2.04 & 1.75 & 2.03 & 2.07 & $<0.0001$ \\
\hline Median & 2 & 2 & 2 & 2 & \\
\hline \multicolumn{6}{|l|}{ DBS indications, $n(\%)$} \\
\hline Essential tremor & $7373(21.9)$ & $506(29.3)$ & $3420(22.2)$ & $3447(20.9)$ & $<0.0001$ \\
\hline PD & $24,787(73.7)$ & $1209(70.0)$ & $11,325(73.5)$ & $12,253(74.2)$ & $<0.0001$ \\
\hline Dystonia & $1482(4.4)$ & $13(0.8)$ & 667 (4.3) & $802(4.9)$ & $<0.0001$ \\
\hline \multicolumn{6}{|l|}{ LOS in days } \\
\hline Mean & 2.0 & 1.95 & 1.93 & 2.89 & $<0.0001$ \\
\hline Median & 1 & 1 & 1 & 1 & \\
\hline \multicolumn{6}{|c|}{$\begin{array}{l}\text { CAD = coronary artery disease; } C H F=\text { congestive heart disease; COPD = chronic obstructive pulmonary disease; PVD = peripheral vascular } \\
\text { disease. } \\
\text { * Values in boldface are statistically significant. } \\
\text { † As per the HCUP data user agreement, values } \leq 10 \text { for individual discharge records cannot be quantified/tabulated to curtail any risk for } \\
\text { patient identification. } \\
\text { † Inflation adjusted values over the } 10 \text {-year period to } 2013 \text { US } \$ \text { amounts using the national consumer price index calculator (http://www.bls. } \\
\text { gov/data/inflation_calculator.htm). }\end{array}$} \\
\hline
\end{tabular}

ber of DBS procedures performed in the US for 3 primary movement disorders was noted over time from 2540 cases in 2002 to 5222 in $2011(\mathrm{r}=0.658, \mathrm{p}=0.038)$. This increase in DBS procedures was most notably seen for dystonia $(\mathrm{r}=$ $0.852, \mathrm{p}=0.002)$, followed by essential tremor $(\mathrm{r}=0.765, \mathrm{p}$ $=0.010$ ), with a 6.33- and 3.41-fold increase over time, respectively. For PD, an increase of 1.71-fold was observed (r
$=0.511, \mathrm{p}=0.131)$ with 2094 cases in 2002 in comparison with 3575 in 2011. Trends of DBS procedures performed over time and per 100,000 US population for PD, dystonia, and essential tremor are shown in Fig. 6. In LVCs, an exponential rise corresponding to a $463 \%$ increase in the mean hospital charges was observed (from $\$ 24,539$ in 2002 to $\$ 113,531$ in $2011[\mathrm{r}=0.89, \mathrm{p}<0.0001])$, whereas a linear

TABLE 2. Postoperative outcomes in patients undergoing DBS in the US between 2002 and $2011(n=33,642)$

\begin{tabular}{lrcccc}
\hline \multicolumn{1}{c}{ Complication } & Overall & LVCs $(\mathrm{n}=1728)$ & MVCs $(\mathrm{n}=15,412)$ & HVCs ( $=16,502)$ & $\mathrm{p} \mathrm{Value*}$ \\
\hline Mortality & $0.2 \%$ & $0.0 \%$ & $0.1 \%$ & $0.3 \%$ & $\mathbf{0 . 0 0 3}$ \\
\hline Unfavorable discharge & $5.9 \%$ & $7.5 \%$ & $6.8 \%$ & $5.0 \%$ & $<0.0001$ \\
\hline High-end hospital charges & $14.6 \%$ & $12.4 \%$ & $13.2 \%$ & $16.2 \%$ & $<0.0001$ \\
\hline Prolonged LOS (days) & $10.2 \%$ & $9.5 \%$ & $8.2 \%$ & $12.2 \%$ & $<0.0001$ \\
\hline Cardiac complications & $0.4 \%$ & $0.6 \%$ & $0.5 \%$ & $0.3 \%$ & $\mathbf{0 . 0 2 4}$ \\
\hline $\begin{array}{l}\text { Neurological complications including those } \\
\text { arising from a nervous system device }\end{array}$ & $5.5 \%$ & $2.0 \%$ & $5.3 \%$ & $6.0 \%$ & $<0.0001$ \\
\hline $\begin{array}{l}\text { Venous thromboembolism } \\
\text { Wound complications }\end{array}$ & $1.8 \%$ & $1.3 \%$ & $1.5 \%$ & $2.0 \%$ & $<0.0001$ \\
\hline
\end{tabular}

$\mathrm{n}=$ number of patients

* Values in boldface are statistically significant. 
A Unfavorable discharge

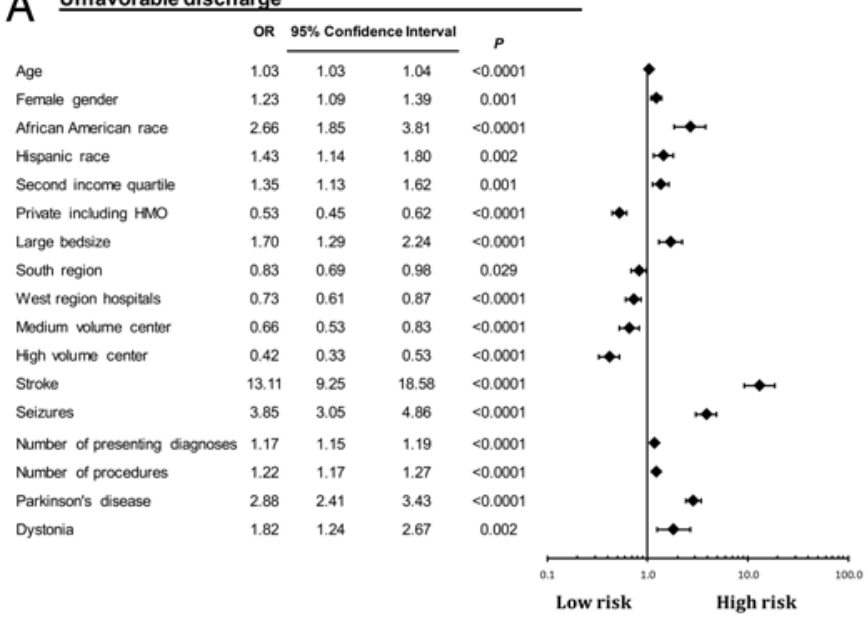

Venous thromboembolism

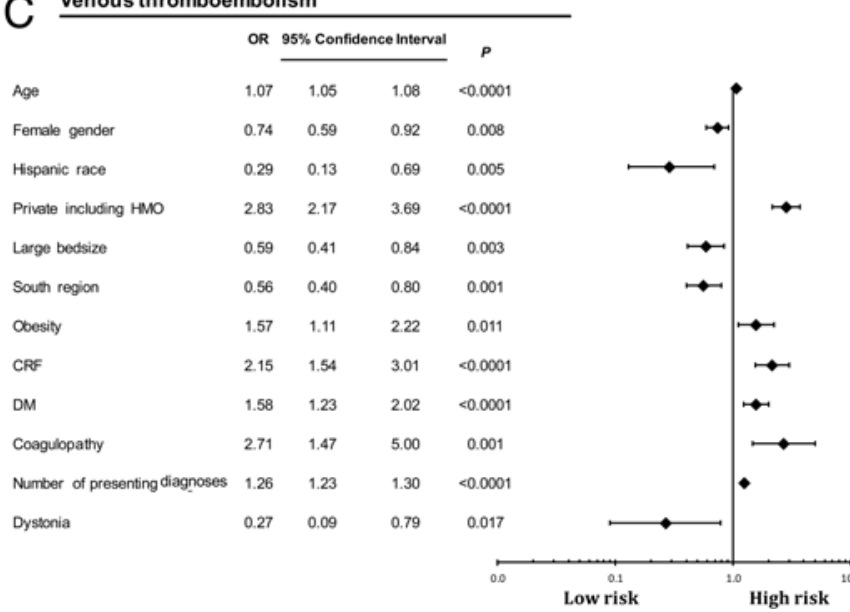

B Cardiac complications
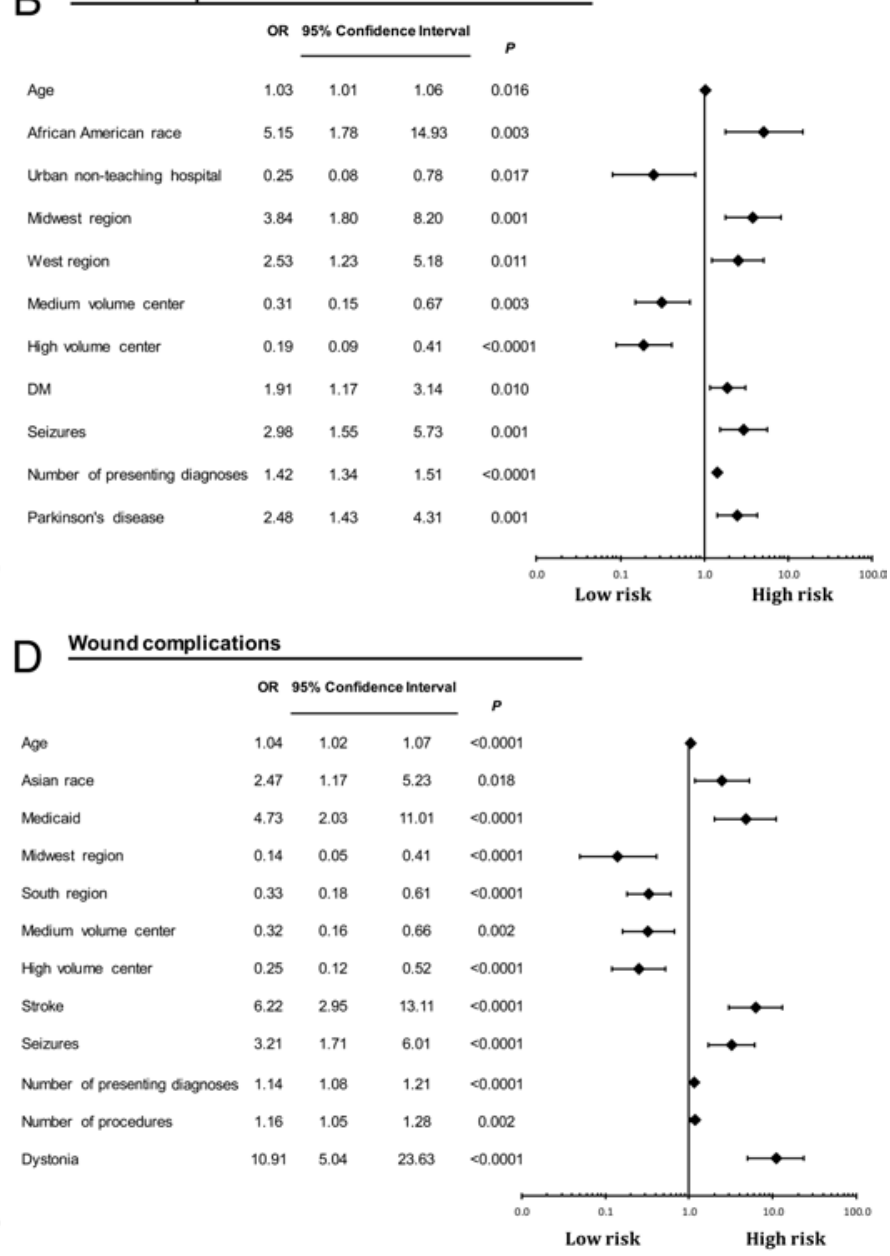

FIG. 3. Multivariable binary logistic regression model demonstrating the association of unfavorable discharge (A), and postoperative cardiac complications (B), wound complications (C), and venous thromboembolism (D) with the exposure variables. The corresponding forest plots are displayed on the right. $\mathrm{HMO}=$ health maintenance organization.

increase approximating a $120 \%$ increase in the mean hospital charges was observed in HVCs $(\$ 54,332$ in 2002 to $\$ 65,267$ in $2011[\mathrm{r}=0.565, \mathrm{p}=0.089]$ ) (Fig. 7). Likewise, a $140 \%$ increase in the mean hospital charges was noted for MVCs $(r=0.576, p=0.081)$ with $\$ 58,430$ in 2002 to $\$ 81,805$ in 2011 . There was a significant difference in the mean charges between HVC and LVC (post hoc test, $\mathrm{p}<$ $0.0001)$.

\section{Discussion}

Deep brain stimulation is a minimally invasive neuromodulation technique that targets specific structures in the brain. Although DBS was described by Benabid et al. ${ }^{6}$ in the 1980s, its popularity increased in the 1990s. Thalamic DBS was approved by the FDA in 1997 for essential tremor. DBS of the subthalamic nucleus and globus pallidus pars internus was approved for PD in 2002. As the number of DBS cases has increased over the years, so have the clinical indications. DBS is now used to treat dystonia, essential tremor, obsessive-compulsive disorder, Tourette's syndrome, epilepsy, cluster headaches, stroke-related pain, phantom-limb pain, trigeminal neuralgia, and multiple sclerosis. $4,21,29,35$

When DBS gained government-regulated approval, an increase in the national number of DBS procedures was noted. In 1996, 756 DBS procedures were performed, which increased to 4200 DBS procedures by $2006 .{ }^{25}$ As DBS became more prevalent, Lad et al. noted that the inpatient LOS declined..$^{25}$ This is likely attributed to centers becoming familiar with new technology and the nuances of patient care required for DBS patients. Unfortunately, there was an increase in hospital charges over the same time period. As beneficial effects of DBS in movement disorders became widespread, the need to establish good practices, better outcomes with minimal adverse events, and enhanced ability to effectively deal with complex cases led to the emergence of multidisciplinary patient management approach, incorporating cross-disciplinary expertise. ${ }^{30,38,40}$ The cross-disciplinary model involved before concluding a case deemed for DBS, in addition to evolving technology, and equipped infrastructure could possibly explain the rise in hospital charges over time. In our analysis, this increase was most notable for LVCs, re- 

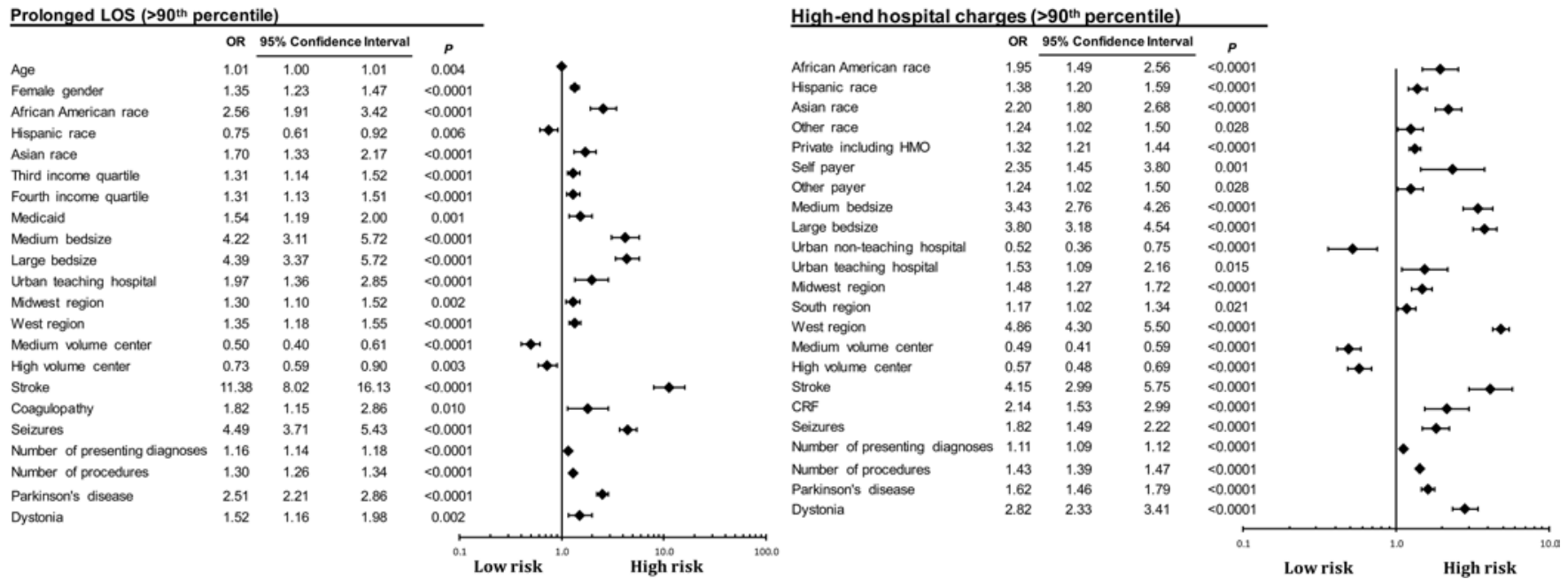

FIG. 4. Multivariable binary logistic regression model demonstrating the association of prolonged LOS (left) and high-end hospital charges (right) with the exposure variables. The corresponding forest plots are displayed on the right.

flecting a 463\% rise in the mean hospital charges in 2011 compared with 2002, despite inflation adjustments. With the same yearly comparisons, we noted that high case volume centers (HVCs and MVCs) were able to significantly contain the rising charges with an observed increase for HVCs and MVCs at $120 \%$ and $140 \%$, respectively, in 2011 from the baseline year 2002. The considerable volume loads in these centers is presumed to have been economical in sustaining a steady increase in charges over time.

Pilitsis et al..$^{36}$ noted a plateau in the number of DBS procedures, which was true from 2004 to 2007. However, our inpatient analysis suggests that since 2006, the number of DBS procedures has continued to increase for $\mathrm{PD}$, essential tremor, and dystonia. This is likely related to several factors. As clinical indications for DBS grow, hospitals are more likely to provide this treatment modality. The equipment needed for DBS procedures has been available for a longer time period now, making it readily available. Because of society's acceptance of DBS and the supporting literature, more patients may inquire about this treatment modality. Because of the demand and popularity, more neurosurgeons are being trained to perform DBS procedures. Initially, when DBS was in the early stages, it was likely to be available only at large academic centers. As more hospitals acquired the equipment and more neurosurgeons were trained in DBS surgery, community hospitals started offering DBS as a treatment modality. The number of hospitals with small- to medium-sized caseloads exceeds large-volume centers significantly, as depicted in Fig. 2.

Several studies have looked at the effects of case volumes at hospitals with regard to complication rates and outcomes. Studies have shown that high caseloads can lead to better outcomes and lower complication rates in subarachnoid hemorrhage, epilepsy surgery, pediatric brain tumor, and other surgical fields., $30,14,16,18,20,41$ One would anticipate complication rates to be greater at LVCs across the board, but this has not been proven in the literature. In fact, the complication rate for DBS has remained small even as more LVCs have arisen. When HVCs and LVCs are compared in our analysis, patients are actually more likely to have a neurological complication arising from an implanted device at an HVC. This is likely as patients with severe comorbidities as stratified by a higher CCI (Table 1) are being treated at an HVC. Since patients with multiple and/or severe comorbidities may have more periopera-
Outcomes in HVCs in comparison to LVCs

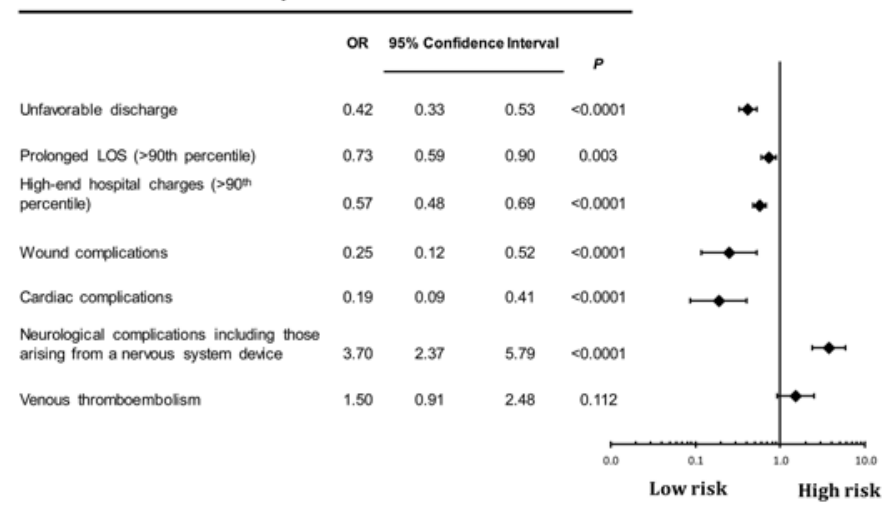

Outcomes in MVCs in comparison to LVCs

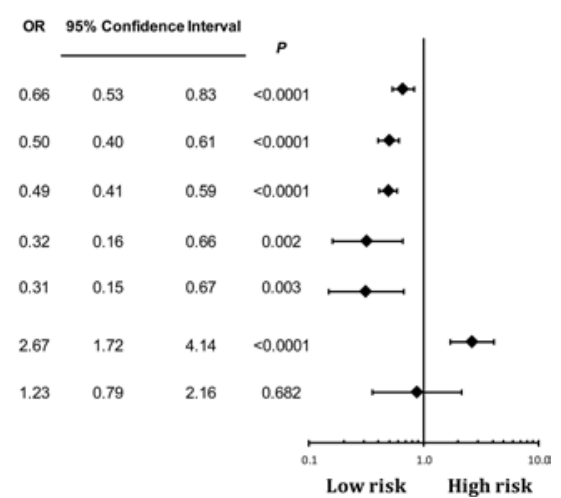

FIG. 5. Multivariable binary logistic regression analysis of unfavorable outcomes in HVCs in comparison with LVCs (left) and MVCs in comparison with LVCs (right). 

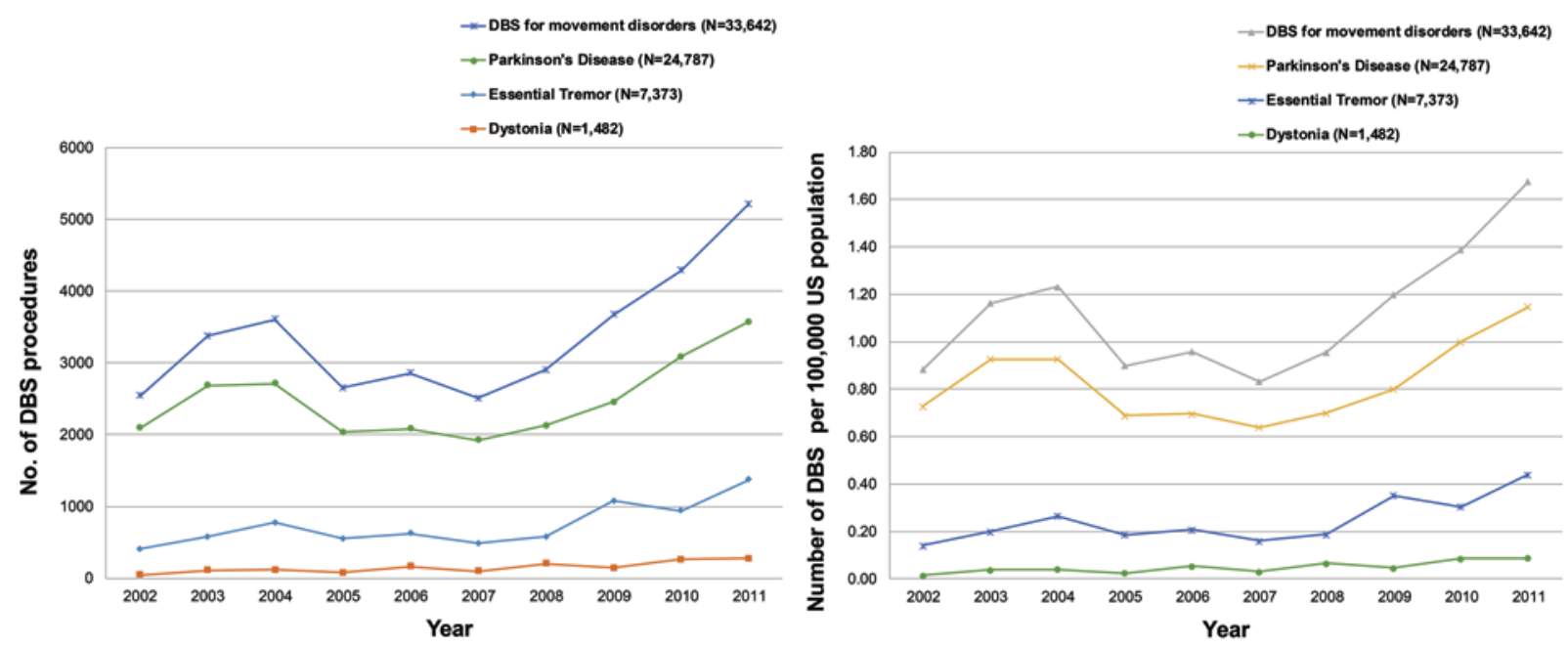

FIG. 6. National estimates of distribution of total number of DBS procedures over time (left) and per 100,000 US population (right).

tive issues, HVCs may appear to have more neurological complications because they are treating sicker patients and more complex cases, which is a limitation of the analysis we performed. If equivocal patients between HVCs and LVCs were analyzed, it is likely that HVCs would exhibit a lower neurological complication rate. As expected, HVCs and MVCs have a lower likelihood of unfavorable discharge, prolonged LOS, high-end hospital charges, cardiac complications, and wound complications (Fig. 5).

In the early days of DBS, it is likely that only the most optimal candidates were selected for surgery. As DBS became more prevalent, the mean age of patients receiving DBS increased from the years 2000 to 2007, and more patients were deemed appropriate candidates despite having comorbidities. Despite patients with higher risk factors undergoing surgery, a $1 \%$ decrease in the complication rate to $2.8 \%$ was noted from 2000 to $2007,{ }^{36}$ probably reflecting the increasing experience of DBS teams. It is also important to

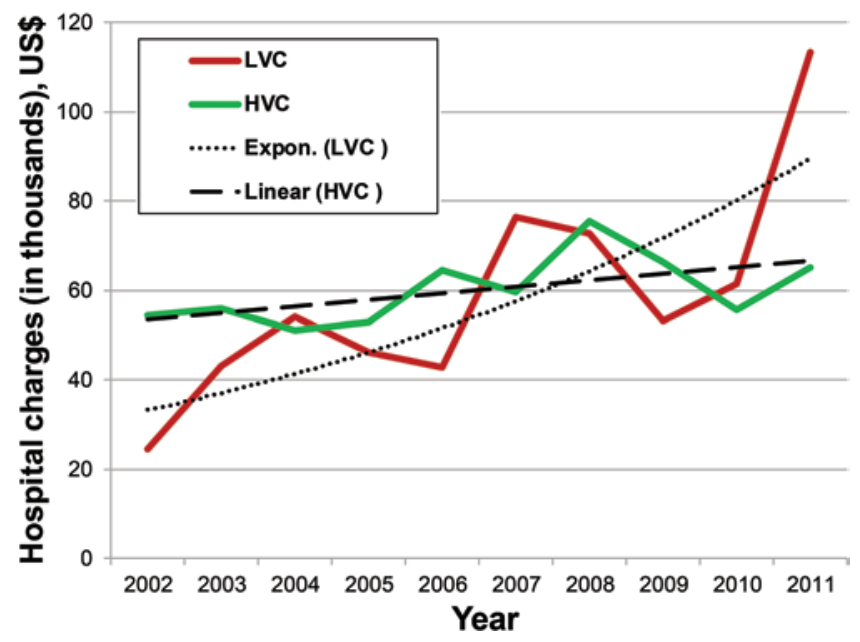

FIG. 7. Trends of hospital charges between HVC and LVC over time. The y axis represents inflation-adjusted 2013 US $\$$ amounts in thousands. The dotted line represents exponential (Expon.) relationship of rise in hospital charges for LVC over time, and the interrupted line represents linear relationship of rise in hospital charges for HVCs over time. note that only the number, not the severity, of comorbidities is quantified in the NIS, which limits the conclusions one can make regarding preexisting patient health and complication rate. Studies have reported poor prognostic factors with regard to DBS surgery. We found several prognostic factors associated with an unfavorable discharge. African American race was found to have a higher risk of an unfavorable discharge. This could be explained by a lower socioeconomic status or the unavailability of routine health care to treat and maintain preexisting medical conditions. Additionally, the second median income quartile (lower income quartile) was found to be independently associated with having higher odds for an unfavorable discharge. Patients with private insurance may have better access to primary care, which could lead to better maintenance of medical conditions. ${ }^{28}$ The lower socioeconomic status of certain population subgroups correlates with low income quartiles having a higher odds risk. Patients with Medicaid were found to have higher odds for prolonged LOS and wound complications, which may be explained by lower baseline levels of healthy living and health care maintenance. A history of remote stroke was by far the greatest risk factor for an unfavorable discharge, prolonged LOS, high-end hospital charges, and wound complications, followed by preexisting seizure disorder. If stroke patients are receiving antiplatelet agents that need to be withheld for surgery, this could contribute to the increased postoperative complication rate. A history of stroke could also lead to an unfavorable discharge, regardless of the hospital course, if one is previously debilitated from the stroke. Patients with a seizure disorder warrant special attention to ensure therapeutic levels of antiepileptic agents, since these patients are more prone to unfavorable discharges. When compared with essential tremor, PD and dystonia are more likely to be associated with unfavorable discharges, prolonged LOS, and high-end hospital charges. This is an expected outcome as PD and dystonia are more debilitating disease processes in general, necessitating ancillary care. Patients with dystonia are also more likely to have wound complications, which is probably because of the extreme physical positions that these patients often maintain perioperatively. 
Eskandar et al. ${ }^{19}$ reported national trends from 1996 to 2000. The authors found that short-term outcomes were superior at hospitals with larger case volumes and correlated with lower mortality rates. Although all centers are achieving relatively low rates of post-DBS complications as the procedure and patient selection become more standardized, the exponential rise in cost in LVCs suggests that decentralization of DBS may not necessarily be a cost-effective solution for the health care system. Supplementing that of Eskandar et al., our analysis quantified the extent of risk of unfavorable outcomes based on hospital volume loads with HVCs and MVCs potentially having lower odds for adverse outcomes. In particular, we explored associations of independent sociodemographics, hospital characteristics, and patient comorbidities on short-term adverse events that could potentially be applied as risk stratification and decision making for patients undergoing DBS for movement disorders.

Despite the well-described limitations of the NIS database, the database does provide a comprehensive sampling of the entire US nonfederal hospital community. It allows for assessing trends and outcomes for numerous diseases, avoids selection bias and provides the largest representative sample (20\% of all inpatient admissions) to estimate inpatient averages and hospital case volumes. Limitations related to the NIS database are well known and are similar to those governing analysis of large administrative databases. $^{26}$ These include coding errors, under-reporting of events, ${ }^{8,32}$ and lack of longitudinal follow-up and outpatient data restricting ability to assess long-term outcomes, including postdischarge data and complications. Inability to stratify medical conditions and comorbidities based on their severity and broad-based definition of diseases and procedures described using the ICD-9-CM codes potentially serves as a confounding factor and were major limitations in our study. Nevertheless, the sheer volume of inpatient data collected prospectively over time and incorporated from various clinical practice settings makes NIS the largest available inpatient database in the US, enabling a generalized overview of outcome assessments for various diseases by minimizing selection bias.

\section{Conclusions}

The NIS is a prospective hospital discharge database containing a representative sample of inpatient admissions to nonfederal hospitals in the US. Using this database, we identified risk factors associated with unfavorable discharge, prolonged LOS, high-end hospital charges, wound and cardiac complications, and venous thromboembolism in patients undergoing DBS for PD, dystonia, and essential tremor. In terms of outcomes following DBS, HVCs and MVCs fare better than LVCs, and quantification of the risks of an adverse event based on hospital case volume is estimated in our study. Although the association of these independent risk factors and the effect of hospital case volume with outcomes could potentially be useful in risk stratification and guide surgical decision making, generalization should be made with caution.

\section{References}

1. Agency for Healthcare Research and Quality: Clinical Clas- sifications Software (CCS) for ICD-9-CM. Healthcare and Utilization Project. (http://www.hcup-us.ahrq.gov/toolssoft ware/ccs/ccs.jsp) [Accessed April 7, 2015]

2. Agency for Healthcare Research and Quality: Overview of the Nationwide Inpatient Sample (NIS). Healthcare and Utilization Project. (http://www.hcup-us.ahrq.gov/nisoverview. jsp) [Accessed April 7, 2015]

3. Bach PB, Cramer LD, Schrag D, Downey RJ, Gelfand SE, Begg CB: The influence of hospital volume on survival after resection for lung cancer. N Engl J Med 345:181-188, 2001

4. Baker KB, Kopell BH, Malone D, Horenstein C, Lowe M, Phillips MD, et al: Deep brain stimulation for obsessivecompulsive disorder: using functional magnetic resonance imaging and electrophysiological techniques: technical case report. Neurosurgery 61:E367-E368, 2007

5. Benabid AL, Pollak P, Gervason C, Hoffmann D, Gao DM, Hommel M, et al: Long-term suppression of tremor by chronic stimulation of the ventral intermediate thalamic nucleus. Lancet 337:403-406, 1991

6. Benabid AL, Pollak P, Louveau A, Henry S, de Rougemont J: Combined (thalamotomy and stimulation) stereotactic surgery of the VIM thalamic nucleus for bilateral Parkinson disease. Appl Neurophysiol 50:344-346, 1987

7. Benabid AL, Pollak P, Seigneuret E, Hoffmann D, Gay E, Perret J: Chronic VIM thalamic stimulation in Parkinson's disease, essential tremor and extra-pyramidal dyskinesias. Acta Neurochir Suppl (Wien) 58:39-44, 1993

8. Berthelsen CL: Evaluation of coding data quality of the HCUP National Inpatient Sample. Top Health Inf Manage 21:10-23, 2000

9. Bhatia S, Oh M, Whiting T, Quigley M, Whiting D: Surgical complications of deep brain stimulation. A longitudinal single surgeon, single institution study. Stereotact Funct Neurosurg 86:367-372, 2008

10. Birkmeyer JD, Sun Y, Wong SL, Stukel TA: Hospital volume and late survival after cancer surgery. Ann Surg 245:777783,2007

11. Bureau of Labor Statistics: Consumer Price Index Inflation Calculator. (http://www.bls.gov/data/inflation_calculator. htm) [Accessed April 7, 2015]

12. Castrioto A, Lozano AM, Poon YY, Lang AE, Fallis M, Moro E: Ten-year outcome of subthalamic stimulation in Parkinson disease: a blinded evaluation. Arch Neurol 68:1550-1556, 2011

13. Charlson ME, Pompei P, Ales KL, MacKenzie CR: A new method of classifying prognostic comorbidity in longitudinal studies: development and validation. J Chronic Dis 40:373383, 1987

14. Cross DT III, Tirschwell DL, Clark MA, Tuden D, Derdeyn $\mathrm{CP}$, Moran CJ, et al: Mortality rates after subarachnoid hemorrhage: variations according to hospital case volume in 18 states. J Neurosurg 99:810-817, 2003

15. Deyo RA, Cherkin DC, Ciol MA: Adapting a clinical comorbidity index for use with ICD-9-CM administrative databases. J Clin Epidemiol 45:613-619, 1992

16. Dimick JB, Cowan JA Jr, Stanley JC, Henke PK, Pronovost PJ, Upchurch GR Jr: Surgeon specialty and provider volumes are related to outcome of intact abdominal aortic aneurysm repair in the United States. J Vasc Surg 38:739-744, 2003

17. Dowling J: Deep brain stimulation: current and emerging indications. Mo Med 105:424-428, 2008

18. Englot DJ, Ouyang D, Wang DD, Rolston JD, Garcia PA, Chang EF: Relationship between hospital surgical volume, lobectomy rates, and adverse perioperative events at US epilepsy centers. J Neurosurg 118:169-174, 2013

19. Eskandar EN, Flaherty A, Cosgrove GR, Shinobu LA, Barker FG II: Surgery for Parkinson disease in the United States, 1996 to 2000: practice patterns, short-term outcomes, and hospital charges in a nationwide sample. J Neurosurg 99:863-871, 2003 
20. Finks JF, Osborne NH, Birkmeyer JD: Trends in hospital volume and operative mortality for high-risk surgery. $\mathbf{N}$ Engl J Med 364:2128-2137, 2011

21. Henderson JM: Vagal nerve stimulation versus deep brain stimulation for treatment-resistant depression: show me the data. Clin Neurosurg 54:88-90, 2007

22. Hosobuchi Y, Adams JE, Rutkin B: Chronic thalamic stimulation for the control of facial anesthesia dolorosa. Arch Neurol 29:158-161, 1973

23. Kenney C, Simpson R, Hunter C, Ondo W, Almaguer M, Davidson A, et al: Short-term and long-term safety of deep brain stimulation in the treatment of movement disorders. J Neurosurg 106:621-625, 2007

24. Kleiner-Fisman G, Fisman DN, Sime E, Saint-Cyr JA, Lozano AM, Lang AE: Long-term follow up of bilateral deep brain stimulation of the subthalamic nucleus in patients with advanced Parkinson disease. J Neurosurg 99:489-495, 2003

25. Lad SP, Kalanithi PS, Patil CG, Itthimathin P, Batya S, Bronte-Stewart H, et al: Socioeconomic trends in Deep Brain Stimulation (DBS) surgery. Neuromodulation 13:182-186, 2010

26. Lawthers AG, McCarthy EP, Davis RB, Peterson LE, Palmer RH, Iezzoni LI: Identification of in-hospital complications from claims data. Is it valid? Med Care 38:785-795, 2000

27. Liang GS, Chou KL, Baltuch GH, Jaggi JL, Loveland-Jones C, Leng L, et al: Long-term outcomes of bilateral subthalamic nucleus stimulation in patients with advanced Parkinson's disease. Stereotact Funct Neurosurg 84:221-227, 2006

28. Lin N, Popp AJ: Insurance status and patient outcome after neurosurgery. World Neurosurg 76:398-400, 2011

29. Malone DA Jr, Dougherty DD, Rezai AR, Carpenter LL, Friehs GM, Eskandar EN, et al: Deep brain stimulation of the ventral capsule/ventral striatum for treatment-resistant depression. Biol Psychiatry 65:267-275, 2009

30. Ogden-Epker M, Cullum CM: Quantitative and qualitative interpretation of neuropsychological data in the assessment of temporal lobectomy candidates. Clin Neuropsychol 15:183-195, 2001

31. Oh MY, Abosch A, Kim SH, Lang AE, Lozano AM: Longterm hardware-related complications of deep brain stimulation. Neurosurgery 50:1268-1276, 2002

32. O'Malley KJ, Cook KF, Price MD, Wildes KR, Hurdle JF, Ashton CM: Measuring diagnoses: ICD code accuracy. Health Serv Res 40:1620-1639, 2005

33. Pahapill PA, O'Connell B: Long-term follow-up study of chronic deep brain stimulation of the subthalamic nucleus for cervical dystonia. Neuromodulation 13:26-30, 2010

34. Pahwa R, Wilkinson SB, Overman J, Lyons KE: Bilateral subthalamic stimulation in patients with Parkinson disease: long-term follow up. J Neurosurg 99:71-77, 2003

35. Pereira EA, Green AL, Nandi D, Aziz TZ: Deep brain stimulation: indications and evidence. Expert Rev Med Devices 4:591-603, 2007

36. Pilitsis JG, Burrows A, Peters ML, Sargent J, Ng SC, Tseng JF: Changing practice patterns of deep brain stimulation in Parkinson's disease and essential tremor in the USA. Stereotact Funct Neurosurg 90:25-29, 2012
37. Quan H, Sundararajan V, Halfon P, Fong A, Burnand B, Luthi JC, et al: Coding algorithms for defining comorbidities in ICD-9-CM and ICD-10 administrative data. Med Care 43:1130-1139, 2005

38. Sanghera MK, Desaloms JM, Stewart RM: High-frequency stimulation of the subthalamic nucleus for the treatment of Parkinson's disease - a team perspective. J Neurosci Nurs 36:301-311, 2004

39. Siegfried J, Shulman J: Deep brain stimulation. Pacing Clin Electrophysiol 10:271-272, 1987

40. Simon CM: Images and image: technology and the social politics of revealing disorder in a North American hospital. Med Anthropol Q 13:141-162, 1999

41. Smith ER, Butler WE, Barker FG II: Craniotomy for resection of pediatric brain tumors in the United States, 1988 to 2000: effects of provider caseloads and progressive centralization and specialization of care. Neurosurgery 54:553565,2004

42. Steiner C, Elixhauser A, Schnaier J: The healthcare cost and utilization project: an overview. Eff Clin Pract 5:143-151, 2002

44. United States Census Bureau (Population Division): Annual Estimates of the Resident Population for the United States, Regions, States, and Puerto Rico: April 1, 2010 to July 1, 2014 (NST-EST2014-01). Washington, DC: US Census Bureau, 2014

43. United States Census Bureau (Population Division): Intercensal Estimates of the resident Population for the United States, Regions, States, and Puerto Rico: April 1, 2000 to July 1, 2010 (ST-EST00INT-01). Washington, DC: US Census Bureau, 2011

\section{Author Contributions}

Conception and design: Kalakoti. Acquisition of data: Kalakoti. Analysis and interpretation of data: Kalakoti. Drafting the article: Kalakoti, Ahmed, Bollam. Critically revising the article: Kalakoti, Wilden, Nanda. Reviewed submitted version of manuscript: Kalakoti, Ahmed, Wilden, Nanda. Approved the final version of the manuscript on behalf of all authors: Nanda. Statistical analysis: Kalakoti, Missios. Administrative/technical/material support: Nanda, Kalakoti, Wilden. Study supervision: Nanda, Missios, Wilden.

\section{Supplemental Information \\ Online-Only Content}

Supplemental material is available online.

Online supplement. http://thejns.org/doi/suppl/10.3171/2015.3. FOCUS1547.

\section{Correspondence}

Anil Nanda, Department of Neurosurgery, Louisiana State Health Sciences Center-Shreveport, P.O. Box 33932, 1501 Kings Highway, Shreveport, LA 71130-3932. email: ananda@1suhsc.edu. 